\title{
ON A DIOPHANTINE EQUATION OF ANDREJ DUJELLA
}

\author{
Keith R. Matthews, John P. Robertson and Jim White
}

University of Queensland and Australian National University, Australia, National Council on Compensation Insurance, USA and Canberra, Australia

\begin{abstract}
We investigate positive solutions $(x, y)$ of the Diophantine equation $x^{2}-\left(k^{2}+1\right) y^{2}=k^{2}$ that satisfy $y<k-1$, where $k \geq 2$. It has been conjectured that there is at most one such solution for a given $k$.
\end{abstract}

\section{INTRODUCTION}

We consider the diophantine equation

$$
x^{2}-\left(k^{2}+1\right) y^{2}=k^{2},
$$

where $k \geq 2$ and $x \geq 1, y \geq 1$.

In 2009, Andrej Dujella remarked that (1.1) always has the solution $(x, y)=\left(k^{2}-k+1, k-1\right)$ and conjectured that there is at most one positive solution $(x, y)$ with $y<k-1$. We call such a solution an exceptional solution and refer to this conjecture as the unicity conjecture. We have verified the conjecture for $k \leq 2^{50}$, with $23,935,816$ values of $k$ possessing a unique exceptional solution. As pointed out by Professor Dujella, the unicity conjecture implies the $D(-1)$ quadruples conjecture (see Section 17). The unicity conjecture has been proved for $k^{2}+1=p^{n}$ or $2 p^{n}, p$ an odd prime and when $k=p^{2 i+1}$ or $2 p^{2 i+1}$, (no exceptional solutions) and when $k=2 p^{2 i}, p$ an odd prime, where the exceptional solution is $\left(2 p^{3 i}+p^{i}, p^{i}\right)$. See [5].

In section 2 we obtain formulae for the exceptional solutions in terms of solutions $(p, q)$ with $\operatorname{gcd}(p, q)=1$, of the diophantine equation $a p^{2}-b q^{2}=$ $2 k / d$, where $a>2, b>2, \operatorname{gcd}(a, b)=1$ and $a b=k^{2}+1$. Then $p / q$ is either a convergent to the continued fraction expansion of $\sqrt{b / a}$ if $d>1$ (which is the case if $k$ is odd), or a near convergent if $d=1$.

2010 Mathematics Subject Classification. 11D09.

Key words and phrases. Quadratic diophantine equations, continued fractions. 
The continued fraction expansion of $\sqrt{b / a}$ has some interesting properties and in section 5 we state Conjecture 5.3 , which relates the solubility of $a p^{2}-$ $b q^{2}=2 k$ with $\operatorname{gcd}(p, q)=1$, to all the partial quotients being even. Also in section 7 , we state Conjecture 7.1 (i) and (ii) which imply the unicity conjecture.

In section 8, we introduce the idea of a Type 1 exceptional solution $(k, x, y)$, i.e., where $y^{2}+1$ divides either $x+y$ or $x-y$. The Type 1 exceptional solutions where $y$ divides $x$ are easy to describe explicitly, while those where $y$ does not divide $x$, in fact satisfy $\operatorname{gcd}(x, y)=1$ and can also be described explicitly.

In section 15, we show that the exceptional solutions form a forest of trees, each arising from a trivial solution as root node: those with root node $(t, t, 0), t \geq 2$ are the solutions with $\operatorname{gcd}(x, y)=t$, whereas those with root node $\left(t, t^{2}-t+1, t-1\right), t \geq 2$ or $\left(t, t^{2}+t+1, t+1\right), t \geq 1$ are the ones with $\operatorname{gcd}(x, y)=1$.

Finally, by studying an extended version of a table of $p / q$ such as Table 1 and using the On-Line Encyclopedia of Integer Sequences OEIS, we were able to guess some families of exceptional solutions, where $a, b, p, q$ and the continued fraction expansion of $\sqrt{b / a}$ are given explicitly.

\section{The PARAMEters $d, a, b, p, q$}

In this section, we derive formulae for the exceptional solution in terms of parameters $d, a, b, p, q$. The special case of squarefree $k$ was dealt with in $[1, \S 27]$.

Proposition 2.1. Suppose $(x, y)$ satisfies equation $(1.1)$. Let $d=\operatorname{gcd}(x+$ $k, x-k)$ and define $a, b, p, q$ by

$$
\begin{gathered}
a=\operatorname{gcd}\left((x+k) / d, k^{2}+1\right), \quad b=\operatorname{gcd}\left((x-k) / d, k^{2}+1\right), \\
p^{2}=(x+k) / d a, \quad q^{2}=(x-k) / d b .
\end{gathered}
$$

Then $p$ and $q$ are integers and

$$
\begin{aligned}
x & =d\left(a p^{2}+b q^{2}\right) / 2, \quad y=d p q, \\
2 k & =d\left(a p^{2}-b q^{2}\right), \quad \operatorname{gcd}(p, q)=1, \\
a b & =k^{2}+1, \quad \operatorname{gcd}(a, b)=1, \\
k & \text { odd } \Longrightarrow d \text { even. }
\end{aligned}
$$

Proof. (i) $x^{2}-\left(k^{2}+1\right) y^{2}=k^{2}$ implies $(x+k)(x-k)=\left(k^{2}+1\right) y^{2}$. Then with $d=\operatorname{gcd}(x+k, x-k)$, we have $x+k=d u, x-k=d v$, where $\operatorname{gcd}(u, v)=1$. We note that if $k$ is odd, then $x$ is odd and so $d$ is even. Then

$$
d^{2} u v=\left(k^{2}+1\right) y^{2} \text {. }
$$


Let $a=\operatorname{gcd}\left(u, k^{2}+1\right), b=\operatorname{gcd}\left(v, k^{2}+1\right)$. We prove $a b=k^{2}+1$. Clearly $a b$ divides $k^{2}+1$, as $\operatorname{gcd}(a, b)=1$. We have $d$ divides $2 k$. If $d$ is odd, then $d$ divides $k$ and $\operatorname{gcd}\left(d, k^{2}+1\right)=1$. If $d=2 D$, then $D$ divides $k$ and $\operatorname{gcd}\left(D, k^{2}+1\right)=1$. Hence if $k$ is even, $\operatorname{gcd}\left(d, k^{2}+1\right)=1$. In both cases, (2.1) implies $d$ divides $y$ and so $k^{2}+1$ divides $u v$. Finally, assume $k$ is odd. Then $2 D^{2} u v=\frac{\left(k^{2}+1\right)}{2} y^{2}$, so $y=2 z$. Then

$$
u v=\left(k^{2}+1\right)(z / D)^{2}
$$

and $k^{2}+1$ divides $u v$. Hence in all cases, $k^{2}+1$ divides the product $\operatorname{gcd}\left(u, k^{2}+\right.$ 1) $\operatorname{gcd}\left(v, k^{2}+1\right)=a b$.

Now let $R=u / a, S=v / b$. Then

$$
\begin{aligned}
u v & =a b R S=\left(k^{2}+1\right) R S, \\
d^{2} u v & =d^{2}\left(k^{2}+1\right) R S=\left(k^{2}+1\right) y^{2} .
\end{aligned}
$$

Hence $d^{2} R S=y^{2}$, so $y=d Y$ and $R S=Y^{2}$. Then $\operatorname{gcd}(R, S)=1$ gives $R=$ $p^{2}, S=q^{2}, Y=p q$ and $y=d p q$. We note that $\operatorname{gcd}\left(a p^{2}, b q^{2}\right)=\operatorname{gcd}(a R, b S)=$ $\operatorname{gcd}(u, v)=1$. Also

$$
2 x=d(u+v)=d\left(a p^{2}+b q^{2}\right) \text { and } 2 k=d(u-v)=d\left(a p^{2}-b q^{2}\right) .
$$

\section{Some Properties OF EXCEPTIONAL SOLUTIONS}

LEMMA 3.1. If $(x, y)$ is an exceptional solution of (1.1), then $a>2$ and $b>2$. Also $d \neq k$ and $d \neq 2 k$.

Proof. (i) First note that $d=k$ or $2 k$ would imply $k$ divides $x+k$ and hence $k$ divides $x$. This in turn implies $k$ divides $y$, contradicting $y<k-1$.

(ii) Suppose $a=1$. Then $b=k^{2}+1$ and $p^{2}-\left(k^{2}+1\right) q^{2}=2 k / d$. Then $p^{2}>\left(k^{2}+1\right) q^{2}>k^{2}$, so $p>k$ and $y=d p q=p q>k$, which is a contradiction.

(iii) Suppose $b=1$, Then $a=k^{2}+1$ and $\left(k^{2}+1\right) p^{2}-q^{2}=2 k / d$. Then $q^{2}=\left(k^{2}+1\right) p^{2}-2 k / d \geq k^{2}+1-2 k=(k-1)^{2}$, so $q \geq k-1$ and $y=d p q \geq k-1$, which is a contradiction.

The cases $a=2$ and $b=2$ are dealt with similarly.

LEMma 3.2. For an exceptional solution, $p$ and $q$ satisfy the following inequalities:

$$
p^{2}<\left(k^{2}+1\right) / d a, \quad q^{2}<(k-1)^{2} / d b .
$$

Proof. If $(k, x, y)$ is an exceptional solution, then $y<k-1$, so $x<$ $k^{2}-k+1$. Hence

$$
\begin{aligned}
& p^{2}=(x+k) / d a<\left(k^{2}+1\right) / d a, \\
& q^{2}=(x-k) / d b<(k-1)^{2} / d b .
\end{aligned}
$$




\section{Exceptional y ARE SMALL}

Proposition 4.1. If $(x, y)$ is an exceptional solution of (1.1), then

$$
y \leq 2 k-\sqrt{3 k^{2}+4} .
$$

Hence $y<(2-\sqrt{3}) k<0.268 k$.

Proof. Equation (1.1) gives

$$
x^{2}=k^{2} y^{2}+k^{2}+y^{2}=(k y+1)^{2}+(k-y)^{2}-1 .
$$

From $y<k-1$, we have $k-y>1$ and so $x>k y+1$. This gives

$$
(k y+1)^{2}+(k-y)^{2}-1 \geq(k y+2)^{2},
$$

or

$$
y^{2}-4 k y+k^{2}-4 \geq 0,
$$

from which inequality (4.1) immediately follows.

The example $k=30$ with exceptional solution $x=242, y=8$, shows that inequality (4.1) is sharp.

\section{Connections With CONTINUEd FRACtions}

Lemma 5.1. ([6, p. 81]) Suppose $Q_{0}$ divides $D$ and $\sqrt{D} / Q_{0}>1$. Then

$$
\sqrt{D} / Q_{0}=\left[a_{0}, \overline{a_{1}, \ldots, a_{l-1}, 2 a_{0}}\right] .
$$

Let $\left(P_{m}+\sqrt{D}\right) / Q_{m}$ denote the $m$-th complete quotient in the continued fraction expansion and let $A_{m} / B_{m}$ denote the $m$-th convergent. Then we have palindromic symmetries for the three sequences

$$
\begin{aligned}
& a_{1}, a_{2}, \ldots, a_{l-2}, a_{l-1}, \\
& P_{1}, P_{2}, \ldots, P_{l-1}, P_{l}, \\
& Q_{0}, Q_{1}, \ldots, Q_{l-1}, Q_{l} .
\end{aligned}
$$

LEMma 5.2. Consider the equation

$$
a p^{2}-b q^{2}=2 k / d,
$$

where $a, b, k, p, q$ are positive, $D=a b=k^{2}+1, \operatorname{gcd}(a, b)=1=\operatorname{gcd}(p, q)$ and $d$ is even if $k$ is odd.

(i) If $d \geq 2$, then $p / q=A_{m} / B_{m}$, a convergent of $\sqrt{b / a}$. Also

$$
Q_{m+1}=2 k / d
$$

where $m$ is odd.

(ii) If $d=1$, then $p / q=\left(A_{m}+e A_{m-1}\right) /\left(B_{m}+e B_{m-1}\right)$, where $e= \pm 1$. Also

$$
(-1)^{m}\left(Q_{m}-Q_{m+1}+2 e P_{m+1}\right)=2 k .
$$


Proof. Since $a p^{2}-b q^{2}=2 k / d$ implies

$$
p / q-\sqrt{b / a}=\frac{2 k}{d(p \sqrt{a}+q \sqrt{b}) q \sqrt{a}},
$$

we have

$$
0<p / q-\sqrt{b / a}<\frac{2 k}{d(2 q \sqrt{b}) q \sqrt{a}}=\frac{2 k}{2 d q^{2} \sqrt{k^{2}+1}}<\frac{1}{d q^{2}} .
$$

Hence if $d \geq 2$, we have $|p / q-\sqrt{b / a}|<1 / 2 q^{2}$ and hence $p / q=A_{m} / B_{m}$, a convergent to $\sqrt{b / a}$. Also

$$
a A_{m}^{2}-b B_{m}^{2}=(-1)^{m+1} Q_{m+1}=2 k / d,
$$

so $Q_{m+1}=2 k / d$ and $m$ is odd.

If $d=1$, inequality (5.3) gives $|p / q-\sqrt{b / a}|<1 / q^{2}$ and hence by the Worley-Dujella lemma [2], we have

$$
p / q=\left(A_{m}+e A_{m-1}\right) /\left(B_{m}+e B_{m-1}\right),
$$

where $e=0$ or \pm 1 and $m \geq 0$. If $e=0$, then $Q_{m+1}=2 k$. Now $\left(P_{m+1}+\right.$ $\sqrt{D}) / Q_{m+1}$ is reduced, as it is the complete quotient of a purely periodic quadratic irrational ([6, Satz 3.3]). Hence

$$
\left(P_{m+1}+\sqrt{D}\right) / Q_{m+1}>1 \text { and }-1<\left(P_{m+1}-\sqrt{D}\right) / Q_{m+1}<0 .
$$

Hence $\sqrt{D}>P_{m+1}>2 k-\sqrt{D}>k-1$, which implies $P_{m+1}=k$. However $D-P_{m+1}^{2} \equiv 0\left(\bmod Q_{m+1}\right)$, i.e., $k^{2}+1-k^{2} \equiv 0(\bmod 2 k)$, giving the contradiction $1 \equiv 0(\bmod 2 k)$.

Finally,

$$
\begin{aligned}
2 k=a p^{2}-b q^{2} & =a\left(A_{m}+e A_{m-1}\right)^{2}-b\left(B_{m}+e B_{m-1}\right)^{2} \\
& =(-1)^{m}\left(Q_{m}-Q_{m+1}+2 e P_{m+1}\right) .
\end{aligned}
$$

(See $[4$, Lemma 2].)

Conjecture 5.3. Suppose $a>1, b>1, a b=k^{2}+1, \operatorname{gcd}(a, b)=1$, where $k$ is even and that the equation $a p^{2}-b q^{2}=2 k$ has a relatively prime solution $(p, q)$. Then in the continued fraction expansion of $\sqrt{b / a}$, all $Q_{i}$ are odd.

REMARK 5.4. This is equivalent to the $P_{i}$ being even, by the identity $Q_{i} Q_{i-1}=D-P_{i}^{2}([6$, p. 69$])$ and the fact that $k$ is even here, so that $D$ is odd. The evenness of the $P_{i}$ is further equivalent to all partial quotients $a_{i}$ being even, by the identity $P_{i+1}=a_{i} Q_{i}-P_{i}([6$, p. 70$])$.

Table 1 lists the $(k, a, b, d, p / q)$ which correspond to exceptional solutions for $k \leq 1000$ via Proposition 2.1 and Lemma 5.2. 


\begin{tabular}{|c|c|c|c|c|c|c|c|}
\hline$k$ & $x$ & $y$ & $a$ & $\bar{b}$ & $\bar{d}$ & $p / q$ & Type \\
\hline 8 & 18 & 2 & 13 & 5 & 2 & $A_{1} / B_{1}=1 / 1$ & 1 \\
\hline 12 & 17 & 1 & 29 & 5 & 1 & $\left(A_{1}-A_{0}\right) /\left(B_{1}-B_{0}\right)=1 / 1$ & 1 \\
\hline 18 & 57 & 3 & 25 & 13 & 3 & $A_{1} / B_{1}=1 / 1$ & 1 \\
\hline 21 & 47 & 2 & 34 & 13 & 2 & $A_{1} / B_{1}=1 / 1$ & 1 \\
\hline 30 & 242 & 8 & 17 & 53 & 4 & $A_{1} / B_{1}=2 / 1$ & 2 \\
\hline 32 & 132 & 4 & 41 & 25 & 4 & $A_{1} / B_{1}=1 / 1$ & 1 \\
\hline 50 & 255 & 5 & 61 & 41 & 5 & $A_{1} / B_{1}=1 / 1$ & 1 \\
\hline 55 & 123 & 2 & 89 & 34 & 2 & $A_{1} / B_{1}=1 / 1$ & 1 \\
\hline 70 & 99 & 1 & 169 & 29 & 1 & $\left(A_{1}-A_{0}\right) /\left(B_{1}-B_{0}\right)=1 / 1$ & 1 \\
\hline 72 & 438 & 6 & 85 & 61 & 6 & $A_{1} / B_{1}=1 / 1$ & 1 \\
\hline 80 & 253 & 3 & 37 & 173 & 1 & $\left(A_{0}+A_{-1}\right) /\left(B_{0}+B_{-1}\right)=3 / 1$ & 1 \\
\hline 98 & 693 & 7 & 113 & 85 & 7 & $A_{1} / B_{1}=1 / 1$ & 1 \\
\hline 105 & 1893 & 18 & 37 & 298 & 6 & $A_{1} / B_{1}=3 / 1$ & 2 \\
\hline 112 & 3362 & 30 & 193 & 65 & 2 & $A_{3} / B_{3}=3 / 5$ & 2 \\
\hline 119 & 1433 & 12 & 194 & 73 & 2 & $A_{3} / B_{3}=2 / 3$ & 2 \\
\hline 128 & 1032 & 8 & 145 & 113 & 8 & $A_{1} / B_{1}=1 / 1$ & 1 \\
\hline 144 & 322 & 2 & 233 & 89 & 2 & $A_{1} / B_{1}=1 / 1$ & 1 \\
\hline 154 & 487 & 3 & 641 & 37 & 1 & $\left(A_{1}-A_{0}\right) /\left(B_{1}-B_{0}\right)=1 / 3$ & 1 \\
\hline 162 & 1467 & 9 & 181 & 145 & 9 & $A_{1} / B_{1}=1 / 1$ & 1 \\
\hline 200 & 2010 & 10 & 221 & 181 & 10 & $A_{1} / B_{1}=1 / 1$ & 1 \\
\hline 203 & 837 & 4 & 130 & 317 & 2 & $A_{1} / B_{1}=2 / 1$ & 1 \\
\hline 208 & 4373 & 21 & 509 & 85 & 1 & $\left(A_{2}+A_{1}\right) /\left(B_{2}+B_{1}\right)=3 / 7$ & 2 \\
\hline 242 & 2673 & 11 & 265 & 221 & 11 & $A_{1} / B_{1}=1 / 1$ & 1 \\
\hline 252 & 8068 & 32 & 65 & 977 & 8 & $A_{1} / B_{1}=4 / 1$ & 2 \\
\hline 288 & 3468 & 12 & 313 & 265 & 12 & $A_{1} / B_{1}=1 / 1$ & 1 \\
\hline 333 & 1373 & 4 & 853 & 130 & 2 & $A_{1} / B_{1}=1 / 2$ & 1 \\
\hline 338 & 4407 & 13 & 365 & 313 & 13 & $A_{1} / B_{1}=1 / 1$ & 1 \\
\hline 377 & 843 & 2 & 610 & 233 & 2 & $A_{1} / B_{1}=1 / 1$ & 1 \\
\hline 392 & 5502 & 14 & 421 & 365 & 14 & $A_{1} / B_{1}=1 / 1$ & 1 \\
\hline 408 & 577 & 1 & 985 & 169 & 1 & $\left(A_{1}-A_{0}\right) /\left(B_{1}-B_{0}\right)=1 / 1$ & 1 \\
\hline 414 & 2111 & 5 & 101 & 1697 & 1 & $\left(A_{0}+A_{-1}\right) /\left(B_{0}+B_{-1}\right)=5 / 1$ & 1 \\
\hline 418 & 46818 & 112 & 241 & 725 & 4 & $A_{3} / B_{3}=7 / 4$ & 2 \\
\hline 450 & 6765 & 15 & 481 & 421 & 15 & $A_{1} / B_{1}=1 / 1$ & 1 \\
\hline 495 & 24755 & 50 & 101 & 2426 & 10 & $A_{1} / B_{1}=5 / 1$ & 2 \\
\hline 512 & 8208 & 16 & 545 & 481 & 16 & $A_{1} / B_{1}=1 / 1$ & 1 \\
\hline 546 & 4402 & 8 & 1237 & 241 & 4 & $A_{1} / B_{1}=1 / 2$ & 2 \\
\hline 578 & 9843 & 17 & 613 & 545 & 17 & $A_{1} / B_{1}=1 / 1$ & 1 \\
\hline 612 & 64263 & 105 & 865 & 433 & 3 & $A_{3} / B_{3}=5 / 7$ & 2 \\
\hline 616 & 3141 & 5 & 3757 & 101 & 1 & $\left(A_{1}-A_{0}\right) /\left(B_{1}-B_{0}\right)=1 / 5$ & 1 \\
\hline 648 & 11682 & 18 & 685 & 613 & 18 & $A_{1} / B_{1}=1 / 1$ & 1 \\
\hline 684 & 2163 & 3 & 949 & 493 & 3 & $A_{1} / B_{1}=1 / 1$ & 1 \\
\hline 697 & 8393 & 12 & 505 & 962 & 2 & $A_{1} / B_{1}=3 / 2$ & 2 \\
\hline 722 & 13737 & 19 & 761 & 685 & 19 & $A_{1} / B_{1}=1 / 1$ & 1 \\
\hline 737 & 4483 & 6 & 290 & 1873 & 2 & $A_{1} / B_{1}=3 / 1$ & 1 \\
\hline 800 & 16020 & 20 & 841 & 761 & 20 & $A_{1} / B_{1}=1 / 1$ & 1 \\
\hline 858 & 61782 & 72 & 145 & 5077 & 12 & $A_{1} / B_{1}=6 / 1$ & 2 \\
\hline 882 & 18543 & 21 & 925 & 841 & 21 & $A_{1} / B_{1}=1 / 1$ & 1 \\
\hline 968 & 21318 & 22 & 1013 & 925 & 22 & $A_{1} / B_{1}=1 / 1$ & 1 \\
\hline 987 & 2207 & 2 & 1597 & 610 & 2 & $A_{1} / B_{1}=1 / 1$ & 1 \\
\hline
\end{tabular}

TABLE 1. Exceptional solutions $(k, x, y), k \leq 1000$.

\section{ON THE CONTINUED FRACTION EXPANSION OF $\sqrt{b / a}$}

Lemma 6.1. Let $\sqrt{b / a}=\left[a_{0}, \overline{a_{1}, \ldots, a_{l-1}, 2 a_{0}}\right]$, where $b>a$ and $\operatorname{gcd}(a, b)=1$. Then

$$
\begin{aligned}
b B_{l-1} & =a\left(a_{0} A_{l-1}+A_{l-2}\right), \\
A_{l-1} & =a_{0} B_{l-1}+B_{l-2} .
\end{aligned}
$$

In particular, a divides $B_{l-1}$. 
Proof.

$$
\begin{aligned}
\sqrt{b / a} & =\left[a_{0}, \ldots, a_{l-1}, 2 a_{0}+\left(\sqrt{b / a}-a_{0}\right)\right] \\
& =\left[a_{0}, \ldots, a_{l-1}, a_{0}+\sqrt{b / a}\right] \\
& =\frac{A_{l-1}\left(a_{0}+\sqrt{b / a}\right)+A_{l-2}}{B_{l-1}\left(a_{0}+\sqrt{b / a}\right)+B_{l-2}} .
\end{aligned}
$$

The desired result then follows by cross-multiplying and equating corresponding coefficients.

Lemma 6.2. Suppose $1<a<b, \operatorname{gcd}(a, b)=1, a b=k^{2}+1, D=a b$. Then

(i) The period-length $l$ of $\sqrt{b / a}$ is odd.

(ii) $A_{l-1} / B_{l-1}=k / a$.

(iii) $A_{l-2} / B_{l-2}=\left(b-k a_{0}\right) /\left(k-a a_{0}\right)$.

(iv) $A_{l} / B_{l}=\left(b+k a_{0}\right) /\left(k+a a_{0}\right)$.

Proof. Let $(x, y)=(k, a)$. Then $\operatorname{gcd}(k, a)=1$ and

$$
a x^{2}-b y^{2}=a\left(k^{2}-a b\right)=a\left(k^{2}-\left(k^{2}+1\right)\right)=-a .
$$

A standard argument shows that $x / y$ is a convergent $k / a=A_{t-1} / B_{t-1}$ of $\sqrt{b / a}$. Then $a A_{t-1}^{2}-b B_{t-1}^{2}=(-1)^{t} Q_{t}=-a, Q_{t}=a$ and $t$ is odd. Then $D B_{t-1}=\left(A_{t-1} P_{t}+A_{t-2} Q_{t}\right) Q_{0}$ by $[6$, p. 70]. This gives

$$
a b=k P_{t}+A_{t-2} a \text {. }
$$

Hence $a$ divides $k P_{t}$ and so $a$ divides $P_{t}$. Suppose $P_{t}=a P$. Then as $\xi_{t}=$ $\left(P_{t}+\sqrt{D}\right) / Q_{t}=P+(\sqrt{D}) / a$ is reduced, we have $P=\lfloor(\sqrt{D}) / a\rfloor=a_{0}$. So $\xi_{t}=a_{0}+\xi_{0}$ and we have found a period for $(\sqrt{D}) / a$ of length $t$. Let $l$ be the least period-length. Then $l \leq t$. Also by Lemma 6.1, $a=B_{t-1}$ divides $B_{l-1}$ and so $t \leq l$. Consequently $l=t$ and hence $l$ is odd.

Next, from (6.3), we have $b=k a_{0}+A_{t-2}$, so $A_{t-2}=b-k a_{0}$. Also from [6, p.70], $P_{t} B_{t-1}=A_{t-1} Q_{0}-Q_{t} B_{t-2}$, so $P_{t}=a a_{0}=k-B_{t-2}$ and hence $B_{t-2}=k-a a_{0}$. Finally,

$$
\begin{aligned}
& A_{l}=a_{l} A_{l-1}+A_{l-2}=2 a_{0} k+\left(b-k a_{0}\right)=b+k a_{0}, \\
& B_{l}=a_{l} B_{l-1}+B_{l-2}=2 a_{0} a+\left(k-a a_{0}\right)=k+a a_{0} .
\end{aligned}
$$

The next result narrows down the search for $p$ and $q$, which correspond to an exceptional solution.

Corollary 6.3. Let $l$ be the period length of the continued fraction expansion for $\sqrt{b / a}$, where $a b=k^{2}+1, \operatorname{gcd}(a, b)=1$ and $1<a<b$.

(i) If $d>1$, then $p / q=A_{m} / B_{m}$, where $m \leq l-2$.

(ii) If $d=1$, then $p / q=\left(A_{m}+e A_{m-1}\right) /\left(B_{m}+e B_{m-1}\right)$,e $= \pm 1$, where $m \leq l-1$. 
Proof. From Lemma 3.2, we have $p^{2}<\left(k^{2}+1\right) / 2 d$.

(i) If $d>1$, we know from Lemma 5.2 that $p / q=A_{m} / B_{m}$ and $p^{2}<$ $\left(k^{2}+1\right) / 4<k^{2}$. Hence $A_{m}=p<k=A_{l-1}$ and so $m<l-1$.

(ii) If $d=1$, Lemma 5.2 implies $p / q=\left(A_{m}+e A_{m-1}\right) /\left(B_{m}+e B_{m-1}\right)$. If $e=1$, then $p=A_{m}+A_{m-1}<A_{l-1}$ and so $A_{m}<A_{l-1}$, as before. If $e=-1$, then $p=A_{m}-A_{m-1} \geq A_{m-2}$, and $m-2<l-1$. Hence $m \leq l$. But $m=l$ implies $p=A_{l}-A_{l-1}=\left(b+k a_{0}\right)-k \geq b>k$, which contradicts the inequality $p^{2}<\left(k^{2}+1\right) / 2$. Hence $m \leq l-1$.

7. EXPERIMENTAL RESUlts FOR $a p^{2}-b q^{2}=2 k / d, \operatorname{gcd}(p, q)=1$

Conjecture 7.1. Consider the family of equations $a p^{2}-b q^{2}= \pm 2 k / d$, where $d$ divides $2 k$ (with d even if $k$ is odd), $\operatorname{gcd}(a, b)=1, D=a b=k^{2}+1,2<$ $a<b$.

(i) Then there is at most one $(a, b, d)$ for which solubility occurs with $\operatorname{gcd}(p, q)=1$.

(ii) Let $\left(p_{0}, q_{0}\right)$ and $\left(p_{1}, q_{1}\right)$ be the least and second least positive solutions. Then $d p_{0} q_{0}<k-1<d p_{1} q_{1}$.

(iii) Let $a p_{0}^{2}-b q_{0}^{2}=N$. Then there are two classes of primitive solutions for $a p^{2}-b q^{2}=N$ with fundamental solutions $\left( \pm p_{0}, q_{0}\right)$. Also there are two classes of primitive solutions for $a p^{2}-b q^{2}=-N$ with fundamental solutions $\left( \pm p_{1}, q_{1}\right)$.

ExAmple 7.2. (i) $k=8$. Then $k^{2}+1=65$ and only $(a, b, d)=$ $(5,13,2)$ give solubility of $a p^{2}-b q^{2}= \pm 2 k / d$ with $\operatorname{gcd}(p, q)=1$ and $2<a<b, a b=65, \operatorname{gcd}(a, b)=1$.

\begin{tabular}{c|ccc}
$\sqrt{13 / 5}=(0+\sqrt{65}) / 5=[1, \overline{1,1,1,1,2}]$. \\
$m$ & $a_{m}$ & $\left(P_{m}+\sqrt{D}\right) / Q_{m}$ & $A_{m} / B_{m}$ \\
\hline 0 & 1 & $(0+\sqrt{65}) / 5$ & $1 / 1$ \\
1 & 1 & $(5+\sqrt{65}) / 8$ & $2 / 1$ \\
2 & 1 & $(3+\sqrt{65}) / 7$ & $3 / 2$ \\
3 & 1 & $(4+\sqrt{65}) / 7$ & $5 / 3$ \\
4 & 1 & $(3+\sqrt{65}) / 8$ & $8 / 5$ \\
5 & 2 & $(5+\sqrt{65}) / 5$ & $21 / 13$ \\
\hline
\end{tabular}

From the first period

$$
\begin{aligned}
& 5 A_{0}^{2}-13 B_{0}^{2}=(-1)^{1} Q_{1}=-8, \\
& 5 A_{3}^{2}-13 B_{3}^{2}=(-1)^{4} Q_{4}=8 .
\end{aligned}
$$

Then $\left(p_{0}, q_{0}\right)=\left(A_{0}, B_{0}\right)=(1,1)$ is the smallest primitive solution of $5 p^{2}-13 q^{2}=-8$, while $\left(p_{1}, q_{1}\right)=\left(A_{3}, B_{3}\right)=(5,3)$ is the smallest primitive solution of $5 p^{2}-13 q^{2}=8$. Also $\left(p_{0}, q_{0}\right)$ gives the exceptional solution $\left(x_{0}, y_{0}\right)=(18,2)$ of $x^{2}-65 y^{2}=64$. 
(ii) $k=12$. Here $D=k^{2}+1=145$ and only $(a, b, d)=(5,29,1)$ give solubility of $a p^{2}-b q^{2}= \pm 2 k / d$ with $\operatorname{gcd}(p, q)=1$ and $2<a<b$, $a b=145, \operatorname{gcd}(a, b)=1$.

\begin{tabular}{c|ccc}
\multicolumn{4}{c}{$\sqrt{29 / 5}=(0+\sqrt{145}) / 5=[2, \overline{2,2,4}]}$. \\
$m$ & $a_{m}$ & $\left(P_{m}+\sqrt{D}\right) / Q_{m}$ & $A_{m} / B_{m}$ \\
\hline 0 & 2 & $(0+\sqrt{145}) / 5$ & $2 / 1$ \\
1 & 2 & $(10+\sqrt{145}) / 9$ & $5 / 2$ \\
2 & 2 & $(8+\sqrt{145}) / 9$ & $12 / 5$ \\
3 & 4 & $(10+\sqrt{145}) / 5$ & $53 / 22$ \\
\hline
\end{tabular}

From the first period we read off

$$
\begin{gathered}
5\left(A_{0}-A_{-1}\right)^{2}-29\left(B_{0}-B_{-1}\right)^{2}=(-1)^{0}\left(Q_{0}-Q_{1}-2 P_{1}\right)=-24, \\
5\left(A_{2}+A_{1}\right)^{2}-29\left(B_{2}+B_{1}\right)^{2}=(-1)^{2}\left(Q_{2}-Q_{3}+2 P_{3}\right)=24 .
\end{gathered}
$$

Then $\left(p_{0}, q_{0}\right)=\left(A_{0}-A_{-1}, B_{0}-B_{-1}\right)=(1,1)$ is the smallest primitive solution of $5 p^{2}-29 q^{2}=-24$, while $\left(p_{1}, q_{1}\right)=\left(A_{2}+A_{1}, B_{2}+B_{1}\right)=$ $(17,7)$ is the smallest primitive solution of $5 p^{2}-29 q^{2}=24$. Also $\left(p_{0}, q_{0}\right)$ gives the exceptional solution $\left(x_{0}, y_{0}\right)=(17,1)$ of $x^{2}-145 y^{2}=144$.

\section{Type 1 And Type 2 eXCeptional solutions}

We can rewrite equation (1.1) as

$$
x^{2}-y^{2}=\left(y^{2}+1\right) k^{2} .
$$

DEFinition 8.1. If $(x, y)$ is an exceptional solution of (1.1) such that

$$
x \equiv \epsilon y \quad\left(\bmod y^{2}+1\right),
$$

where $\epsilon= \pm 1$, we call $(x, y)$ a Type 1 solution of (1.1). Any other exceptional solution is called a Type 2 solution.

In the range $2 \leq k \leq 1000$, there are 37 Type 1 and 12 Type 2 exceptional solutions (see Table 1 ) while in the range $2 \leq k \leq 2^{50}$, there are 23, 862, 782 Type 1 and 73,034 Type 2 exceptional solutions.

\section{Exceptional SOlutions Where $y$ Divides $x$.}

It is easy to derive formulae for $k$ and $x$ in terms of $y$, when $y$ divides $x$.

ThEOREM 9.1. Suppose $(x, y)$ is an exceptional solution of (1.1) such that $y$ divides $x$. Then

$$
x+k \sqrt{y^{2}+1}=y\left(2 y^{2}+1+2 y \sqrt{y^{2}+1}\right)^{n},
$$

where $n y>1$. Conversely if $k, x, y$ satisfy (9.1) where ny $>1$, then $(x, y)$ is an exceptional solution of (1.1) with $y$ dividing $x$. 
Proof. If $(x, y)$ is a solution of (1.1) such that $y$ divides $x$, then we see that $y^{2}$ divides $k^{2}$ and hence $y$ divides $k$. From (1.1) we have

$$
(x / y)^{2}-\left(y^{2}+1\right)(k / y)^{2}=1 .
$$

This is a Pell equation whose positive solutions $(x / y, k / y)$ are given by

$$
(x / y)+(k / y) \sqrt{y^{2}+1}=\left(2 y^{2}+1+2 y \sqrt{y^{2}+1}\right)^{n}, n \geq 1 .
$$

Hence (9.1) holds.

Suppose $n=1$. Then $k=2 y^{2}>y+1$ and hence $y>1$. Consequently $n y>1$.

Conversely, assume $k, x, y$ satisfy (9.1), where $n y>1$. Then

$$
x-k \sqrt{y^{2}+1}=y\left(2 y^{2}+1-2 y \sqrt{y^{2}+1}\right)^{n} .
$$

Multiplying corresponding sides of (9.1) and (9.3) gives

$$
x^{2}-k^{2}\left(y^{2}+1\right)=y^{2}\left(\left(2 y^{2}+1\right)^{2}-(2 y)^{2}\left(y^{2}+1\right)\right)^{n}=y^{2},
$$

so $(x, y)$ satisfies (1.1). Also the formula

$$
x=y \sum_{\substack{i=0 \\
i \text { even }}}^{n}\left(\begin{array}{l}
n \\
i
\end{array}\right)\left(2 y^{2}+1\right)^{n-i}(2 y)^{i}\left(y^{2}+1\right)^{i / 2}
$$

reveals that $y$ divides $x$. Hence $y$ divides $k$ and $y \leq k$. But we cannot have $y=k$ as this gives $x^{2}=k^{4}+2 k^{2}$ and so $\left(k^{2}+1\right)^{2}-x^{2}=1$. Hence $\left(k^{2}+1+x\right)\left(k^{2}+1-x\right)=1$, which clearly gives a contradiction. Also $y=k-1$ implies $k-1$ divides $k$, so $k=2, y=1, x=3$. Then (9.1) becomes

$$
3+2 \sqrt{5}=(3+2 \sqrt{5})^{n},
$$

which implies $n=1$ and hence $n y=1$.

EXAMPLE 9.2. (a) $n=1, y>1$ gives $x=2 y^{3}+y$ and $k=2 y^{2}$, an example in [5], where it was proved that the exceptional solution $(x, y)$ is unique if $y$ is a prime.

(b) $n=2$ gives $x=8 y^{5}+8 y^{3}+y$ and $k=8 y^{4}+4 y^{2}$.

TheOREM 9.3. The solutions $(x, y)$ given by (9.1) are of Type 1.

Proof. On considering $(9.4)\left(\bmod y^{2}+1\right)$, only the term $i=0$ remains and we get

$$
x \equiv(-1)^{n} y \quad\left(\bmod y^{2}+1\right),
$$

showing that $(x, y)$ is a Type 1 solution. 


\section{The Structure of Type 1 exceptional solutions}

LEMMA 10.1. There is a one-to-one correspondence between the Type 1 solutions $(x, y), x \equiv \epsilon y\left(\bmod y^{2}+1\right), \epsilon= \pm 1$ and integer pairs $(r, s)$ which satisfy $1<r<s$ and

$$
\begin{aligned}
r^{2}+s^{2} & =k^{2}+1, \\
s & \equiv \epsilon \quad(\bmod r),
\end{aligned}
$$

given by

$$
r=\frac{x-\epsilon y}{y^{2}+1}, \quad s=\frac{x y+\epsilon}{y^{2}+1}
$$

where we take $\epsilon=1$ if $y=1$. The inverse is given by the equations

$$
\begin{aligned}
& x=r+y s, \\
& s=y r+\epsilon .
\end{aligned}
$$

REMARK 10.2. It follows that the unicity conjecture implies that $k^{2}+1$ is expressible in at most one way as $r^{2}+s^{2}$, where $1<r<s, \operatorname{gcd}(r, s)=1$ and $r$ divides $s \pm 1$.

Proof. Assume $(x, y)$ is a Type 1 solution and that $(r, s)$ is given by (10.3). Then it is easy to check that (10.1), (10.2), (10.4) and (10.5) hold.

(i) Clearly $x>y$, hence $r>0$. But $r=1$ implies $y^{2}+1=x-\epsilon y, x=$ $\epsilon y+y^{2}+1$. Then equation (1.1) implies $\left(\epsilon y+y^{2}+1\right)^{2}-\left(y^{2}+1\right) k^{2}=y^{2}$, which gives

$$
\left(y^{2}+1\right)\left(y^{2}+1+2 \epsilon y-k^{2}\right)=0 .
$$

Hence $y^{2}+1+2 \epsilon y-k^{2}=0$, so $(y+\epsilon)^{2}=k^{2}$ and $y+\epsilon=k$, a contradiction, as $y \leq k-2$. Hence $r>1$.

(ii) We have the equivalence

$$
r<s \Longleftrightarrow x-\epsilon y<x y+\epsilon \Longleftrightarrow-\epsilon(y+1)<x(y-1) .
$$

Case 1. Assume $y>1$. Then

$$
x^{2}=k^{2} y^{2}+y^{2}+k^{2}>y^{2}+2 y+1=(y+1)^{2},
$$

so $x>y+1$. Hence $x(y-1)>(y+1)(y-1) \geq y+1$ and (10.6) implies $r<s$.

Case 2. Assume $y=1$. Then $r=(x-1) / 2<(x+1) / 2=s$. 
Conversely, assume $r^{2}+s^{2}=k^{2}+1$, where $s \equiv \epsilon(\bmod r)$ and $1<r<s$. With $y$ defined by $s=y r+\epsilon$ and $x=r+y s$, we have

$$
\begin{aligned}
x^{2}-\left(k^{2}+1\right) y^{2} & =(r+y s)^{2}-\left(r^{2}+s^{2}\right) y^{2} \\
& =r^{2}+2 s r y-r^{2} y^{2} \\
& =r^{2}+2 s(s-\epsilon)-(s-\epsilon)^{2} \\
& =r^{2}+s^{2}-1=k^{2} .
\end{aligned}
$$

Also $x-y \epsilon=(r+y s)-y \epsilon=r+y^{2} r=r\left(1+y^{2}\right)$ and it follows that $(x, y)$ is a Type 1 solution to (1.1).

Finally we have to prove $y<k-1$, or $(s-\epsilon) / r<k-1$. We have $s<k$. Hence $s-\epsilon \leq k$ and $(s-\epsilon) / r \leq k / 2<k-1$.

Lemma 10.3. Assume $(x, y)$ is a Type 1 solution with $\operatorname{gcd}(x, y)=1$. Then $x$ is odd.

(i) If $y$ is even, then $r=u^{2}$, where $u$ is odd and $k=u v$, where $\operatorname{gcd}(u, v)=$ 1.

(ii) If $y$ is odd and $x+\epsilon y \equiv 0(\bmod 4)$, then $r=u^{2}$, where $u$ is odd, $k=u v, v$ is even and $\operatorname{gcd}(u, v)=1$.

(iii) If $y$ is odd and $x+\epsilon y \equiv 2(\bmod 4)$, then $r=2 u^{2}$, where $u$ is odd, $k=u v, v$ is even and $\operatorname{gcd}(u, v)=1$.

Proof. Assume $(x, y)$ satisfies $\operatorname{gcd}(x, y)=1$ and is a Type 1 exceptional solution. If $y$ is even, then $x$ is odd. Also if $y$ is odd, the equation $x^{2}=$ $y^{2}+\left(y^{2}+1\right) k^{2}$ shows $x$ is odd.

Now let $d=\operatorname{gcd}(x-y, x+y)$. Then $d=1$ if $y$ is even, while $d=2$ if $y$ is odd.

We have

$$
\left(\frac{x-\epsilon y}{y^{2}+1}\right)(x+\epsilon y)=k^{2} .
$$

(i) Assume $y$ is even. Then (10.7) gives $r=u^{2}, x+\epsilon y=v^{2}$, where $\operatorname{gcd}(u, v)=$ 1 and $k=u v$.

Assume $y$ is odd. Then $x-\epsilon y=2 X, x+\epsilon y=2 Y$, with $\operatorname{gcd}(X, Y)=1$. Then

$$
\left(\frac{X}{\left(y^{2}+1\right) / 2}\right)(2 Y)=k^{2}
$$

and $k=2 K$ say. Hence

$$
\left(\frac{X}{\left(y^{2}+1\right) / 2}\right) Y=2 K^{2} .
$$

(ii) Assume $x+\epsilon y \equiv 0(\bmod 4)$. Then $Y$ is even, $Y=2 V^{2}, X$ is odd and $r=X /\left(\left(y^{2}+1\right) / 2\right)=u^{2}$, where $u$ is odd, $k=2 u V=u v$, where $v$ is even and $\operatorname{gcd}(u, v)=1$. 
(iii) Assume $x+\epsilon y \equiv 2(\bmod 4)$. Then $Y$ is odd, $Y=V^{2}, X / 2$ is odd and

$$
r / 2=(X / 2) /\left(\left(y^{2}+1\right) / 2\right)=u^{2},
$$

where $u$ is odd. Then $r=2 u^{2}, k=2 u V=u v$, where $u$ is odd, $v$ is even and $\operatorname{gcd}(u, v)=1$.

11. Type 1 solutions $(x, y)$ HaVe $\operatorname{gcd}(x, y)=y$ OR 1 .

In this section, we prove that if $(x, y)$ is a Type 1 solution for which $y$ does not divide $x$, then $\operatorname{gcd}(x, y)=1$.

LEMmA 11.1. With $r$ defined as in Lemma 10.1, let $h=k-r y$. Then $r>h \geq 0$.

PROOF.

$$
\begin{aligned}
r>h & \Longleftrightarrow r>k-r y \\
& \Longleftrightarrow r(y+1)>k \\
& \Longleftrightarrow r^{2}\left(y^{2}+2 y+1\right)>k^{2}=r^{2}+s^{2}-1=r^{2}+(y r+\epsilon)^{2}-1 \\
& \Longleftrightarrow 2 r y(r-\epsilon)>0 .
\end{aligned}
$$

However $r-\epsilon \geq 1$ and consequently $r>h$.

Also $r y+\epsilon=s \leq k-1$, so $0 \leq 1+\epsilon \leq k-r y=h$.

TheOREM 11.2. For a Type 1 solution $(x, y)$ of $(1.1)$, either $y$ divides $x$ or $\operatorname{gcd}(x, y)=1$.

Proof. We present the proof in the form of an algorithm which terminates by determining that either $y$ divides $x$ or $\operatorname{gcd}(x, y)=1$. First we note that from $x=r+y s$, we have $\operatorname{gcd}(x, y)=\operatorname{gcd}(r, y)$.

Let $r_{0}=r, h_{0}=h$. Then $r_{0}>h_{0} \geq 0$ by Lemma 11.1. Also substituting $s=r y+\epsilon$ and $k=r y+h$ in $r^{2}+s^{2}=k^{2}+1$ gives

$$
r_{0}^{2}-2 r_{0} y\left(h_{0}-\epsilon\right)-h_{0}^{2}=0 .
$$

If $h_{0}=0,(11.1)$ implies $r_{0}\left(r_{0}+2 y \epsilon\right)=0$ and so $r_{0}=-2 y \epsilon$ and $y$ divides $r_{0}$ and hence $x$. Also equation (11.1) implies $r_{0}$ divides $h_{0}^{2}$; so $h_{0}=1$ implies $r_{0}=h_{0}$, contradicting Lemma 11.1. Hence we can now assume $r_{0}>h_{0}>1$ and inductively define $r_{n}$ and $h_{n}$ for $n \geq 0$.

If $n \geq 0,\left|r_{n}\right|>\left|h_{n}\right|>1$ and $r_{n}$ is a root of

$$
P_{n}(R)=R^{2}-2 R y\left(h_{n}-\epsilon\right)-h_{n}^{2},
$$

we define $r_{n+1}$ to be the other root of $P_{n}(R)$ :

$$
r_{n+1}=-r_{n}+2 y\left(h_{n}-\epsilon\right) \text {. }
$$

Then

so $1 \leq\left|r_{n+1}\right|<\left|h_{n}\right|$.

$$
\left|r_{n}\right|\left|r_{n+1}\right|=h_{n}^{2}
$$


If $\left|r_{n+1}\right|=1$, then $r_{n} \equiv \pm 1(\bmod 2 y)$ and by (11.3) it follows inductively that $r_{0} \equiv \pm 1(\bmod 2 y)$. Hence $\operatorname{gcd}\left(r_{0}, y\right)=1$ and so $\operatorname{gcd}(x, y)=1$ and we exit the algorithm. We note for future reference that $r_{0}$ is odd in this case.

If $\left|r_{n+1}\right|>1$, we define the polynomial

$$
Q_{n}(H)=H^{2}+2 r_{n+1} y H-2 r_{n+1} y \epsilon-r_{n+1}^{2} .
$$

Then $Q_{n}\left(h_{n}\right)=0$ and we let $h_{n+1}$ be the other root of $Q_{n}(H)$ :

$$
h_{n+1}=-h_{n}-2 r_{n+1} y .
$$

Then

$$
h_{n+1}^{2}+2 r_{n+1} y h_{n+1}-2 r_{n+1} y \epsilon-r_{n+1}^{2}=0 .
$$

Now $\left|h_{n+1}\right|=1$ implies $\left|r_{n+1}\right|=1$, as by (11.6), $r_{n+1}$ divides $h_{n+1}$. This contradicts our assumption that $\left|r_{n+1}\right|>1$.

Hence $\left|h_{n+1}\right| \neq 1$ and we let $H_{n}=h_{n}-\epsilon$. Then

$$
\begin{aligned}
H_{n} H_{n+1} & =h_{n} h_{n+1}-\epsilon\left(h_{n}+h_{n+1}\right)+1 \\
& =-2 r_{n+1} y \epsilon-r_{n+1}^{2}+2 r_{n+1} y \epsilon+1 \\
& =1-r_{n+1}^{2} .
\end{aligned}
$$

Hence $\left|H_{n}\right|\left|H_{n+1}\right|=r_{n+1}^{2}-1>0$. Also

$$
\left|H_{n}\right|=\left|h_{n}-\epsilon\right| \geq\left|h_{n}\right|-1 \geq\left|r_{n+1}\right| \text {. }
$$

Hence

$$
\left|h_{n+1}-\epsilon\right|=\left|H_{n+1}\right|=\frac{r_{n+1}^{2}-1}{\left|H_{n}\right|} \leq \frac{r_{n+1}^{2}-1}{\left|r_{n+1}\right|}<\left|r_{n+1}\right|,
$$

so $\left|h_{n+1}\right| \leq\left|r_{n+1}\right|$. Assume $\left|h_{n+1}\right|=\left|r_{n+1}\right|$. Then (11.6) implies $h_{n+1}=\epsilon$, which gives the contradiction $\left|r_{n+1}\right|=1$. Hence $\left|h_{n+1}\right|<\left|r_{n+1}\right|$.

If $h_{n+1}=0$, then (11.6) implies $-2 r_{n+1} y \epsilon-r_{n+1}^{2}=0$, so $r_{n+1}=-2 y \epsilon$. Then as $r_{n}+r_{n+1}=2 y\left(h_{n}-\epsilon\right)$, it follows inductively that $r_{0} \equiv 0(\bmod 2 y)$. Then $y$ divides $r_{0}$ and hence $x$ and we exit the algorithm. We note for future reference that in this case, $r_{0}$ is even.

Hence

$$
1<\left|h_{n+1}\right|<\left|r_{n+1}\right|<\left|h_{n}\right|<\left|r_{n}\right|,
$$

so both $\left|h_{n+1}\right|$ and $\left|r_{n+1}\right|$ have strictly decreased.

Eventually the algorithm reaches $\left|r_{i}\right|=1$ or $h_{i}=0$ for some $i \geq 0$ and terminates.

Corollary 11.3. For a Type 1 solution $(x, y)$ of (1.1), if $y>1$ and $\operatorname{gcd}(x, y)=1$, then $r=u^{2}$, where $u$ is odd and $k=u v$, where $\operatorname{gcd}(u, v)=1$ and $v>u>1$. Also

$$
v^{2}-\left(y^{2}+1\right) u^{2}=2 y \epsilon .
$$


Proof. We saw in the proof of Theorem 11.2, that either $r \equiv \pm 1$ $(\bmod 2 y)$ or $r \equiv 0(\bmod 2 y)$. Hence if $\operatorname{gcd}(x, y)=1$ and $y>1$, then $r \equiv x \not \equiv 0$ $(\bmod y)$, so we must have $r \equiv \pm 1(\bmod 2 y)$ and hence $r$ is odd. Then parts (i) and (ii) of Lemma 10.3 give $r=u^{2}$, where $k=u v$ and $\operatorname{gcd}(u, v)=1$. Also

$$
s^{2}-1=k^{2}-r^{2}=u^{2} v^{2}-u^{4} .
$$

Then, as $s>1,(11.8)$ gives $u^{2} v^{2}-u^{4}>0$ and hence $v>u$.

Finally, $s=y r+\epsilon=y u^{2}+\epsilon$, so

$$
s^{2}-1=u^{2}\left(y^{2} u^{2}+2 y \epsilon\right) \text {. }
$$

Then (11.8) and(11.9) give

$$
v^{2}-\left(y^{2}+1\right) u^{2}=2 y \epsilon .
$$

We can now derive explicit formulae for $x, y$ and $k$ for a Type 1 solution $(x, y)$ with $\operatorname{gcd}(x, y)=1$ and $y>1$.

THEOREM 11.4. If $(x, y)$ is a Type 1 exceptional solution of equation (1.1), with $\operatorname{gcd}(x, y)=1$ and $y>1$, then $k=u_{n} v_{n}$, where

(11.10) $v_{n}+u_{n} \sqrt{y^{2}+1}=f\left(g(y+\epsilon)+\sqrt{y^{2}+1}\right)\left(2 y^{2}+1+2 y \sqrt{y^{2}+1}\right)^{n}$, where $n \geq 1$ and one of the following four possibilities holds:

(a) $f=g=1, \epsilon=1$.

(b) $f=g=1, \epsilon=-1$.

(c) $f=1, g=-1, \epsilon=-1$.

(d) $f=g=-1, \epsilon=1$.

Proof. Assume $(x, y)$ is a Type 1 exceptional solution of equation (1.1), with $\operatorname{gcd}(x, y)=1$ and $y>1$. Then by Corollary 11.3 and equation 11.7, $k=u v$, where

$$
v^{2}-\left(y^{2}+1\right) u^{2}=2 y \epsilon .
$$

We now apply Lemma 3.6 of [7]. This was stated with $y>2$ and $\epsilon=-1$, but also holds with $y>1$ and $\epsilon= \pm 1$. We have

$$
v+u \sqrt{y^{2}+1}=f\left(g(y+\epsilon)+\sqrt{y^{2}+1}\right)\left(2 y^{2}+1+2 y \sqrt{y^{2}+1}\right)^{n},
$$

where $f= \pm 1, g= \pm 1, n$ an integer. Clearly $n \neq 0$ as $n=0$ implies $u=1$.

This gives 16 possibilities for $f, g, \epsilon$ and the sign of $n$. We then eliminate all 8 cases where $n<0$. Of the 8 cases where $n \geq 1$, only (a)-(d) remain. See section 12 for the proof of one case with $n>0$ and for one case with $n<0$. 


\section{Two EXAmples of Sign Determination}

(1) We prove that if $n \geq 1$ and $f=1, g=-1, \epsilon=1$, then $v$ and $u$ given by (11.10) satisfy $v<0$ and $u<0$. Let

$$
v_{n}+u_{n} \sqrt{y^{2}+1}=\left(-(y+1)+\sqrt{y^{2}+1}\right)\left(2 y^{2}+1+2 y \sqrt{y^{2}+1}\right)^{n} .
$$

Then $v_{1}=-\left(2 y^{2}-y+1\right)<0, u_{1}=-(2 y-1)<0$. Also

$v_{n+1}=\left(2 y^{2}+1\right) v_{n}+2 y\left(y^{2}+1\right) u_{n}, \quad u_{n+1}=2 y v_{n}+\left(2 y^{2}+1\right) u_{n}$.

It follows by induction on $n \geq 1$ that $v_{n}<0$ and $u_{n}<0$.

(2) We prove that if $n=-N<0$ and $f=1, g=1, \epsilon=1$, then $v$ and $u$ given by (11.10) satisfy $v>0$ and $u<0$. Let $v_{N}^{\prime}=v_{n}, u_{N}^{\prime}=u_{n}$.

$$
v_{N}^{\prime}+u_{N}^{\prime} \sqrt{y^{2}+1}=\left(y+1+\sqrt{y^{2}+1}\right)\left(2 y^{2}+1-2 y \sqrt{y^{2}+1}\right)^{N} .
$$

Then $v_{1}^{\prime}=2 y^{2}-y+1>0, u_{1}^{\prime}=-(2 y-1)<0$. Also

$$
v_{N+1}^{\prime}=\left(2 y^{2}+1\right) v_{N}^{\prime}-2 y\left(y^{2}+1\right) u_{N}^{\prime}, \quad u_{N+1}^{\prime}=-2 y v_{N}^{\prime}+\left(2 y^{2}+1\right) u_{N}^{\prime} .
$$

It follows by induction on $N \geq 1$ that $v_{N}^{\prime}>0$ and $u_{N}^{\prime}<0$.

\section{Removal of parameters $f$ And $g$}

Let $D=y^{2}+1$. Then equation (11.10) with conjugation gives

$$
\begin{aligned}
& v_{n}+u_{n} \sqrt{D}=(a+b \sqrt{D}) \alpha^{n}, \\
& v_{n}-u_{n} \sqrt{D}=(a-b \sqrt{D}) \beta^{n},
\end{aligned}
$$

where $a=f g(y+\epsilon), b=f$ and

$$
\alpha=2 y^{2}+1+2 y \sqrt{D}, \quad \beta=2 y^{2}+1-2 y \sqrt{D} .
$$

Note that $\alpha \beta=1$. First we remove $f$.

Lemma 13.1. If $(k, x, y)$ is a Type 1 exceptional solution satisfying $\operatorname{gcd}(x, y)=1, y>1$, then $(x, k)=\left(x_{n}, k_{n}\right)$, where

$$
x_{n}+k_{n} \sqrt{D}=\left(y^{2}+\epsilon y+1+g(y+\epsilon) \sqrt{D}\right) \alpha^{2 n}, n \geq 1,
$$

and $g= \pm 1, \epsilon= \pm 1$.

Proof. We know that

$$
\begin{aligned}
x_{n}+k_{n} \sqrt{D} & =u_{n}^{2} D+y \epsilon+v_{n} u_{n} \sqrt{D} \\
& =u_{n} \sqrt{D}\left(v_{n}+u_{n} \sqrt{D}\right)+y \epsilon \\
& =\frac{\left((a+b \sqrt{D}) \alpha^{n}-(a-b \sqrt{D}) \beta^{n}\right)}{2}(a+b \sqrt{D}) \alpha^{n}+y \epsilon \\
& =\frac{(a+b \sqrt{D})^{2}}{2} \alpha^{2 n}-\frac{\left(a^{2}-b^{2} D\right)}{2}+y \epsilon \\
& =\frac{(a+b \sqrt{D})^{2}}{2} \alpha^{2 n}
\end{aligned}
$$




$$
\begin{aligned}
& =\frac{(f g(y+\epsilon)+f \sqrt{D})^{2}}{2} \alpha^{2 n} \\
& =\frac{(y+\epsilon)^{2}+2 g(y+\epsilon) \sqrt{D}+y^{2}+1}{2} \alpha^{2 n} \\
& =\left(y^{2}+\epsilon y+1+g(y+\epsilon) \sqrt{D}\right) \alpha^{2 n} .
\end{aligned}
$$

Now we remove $g$.

Corollary 13.2. If $(k, x, y)$ is a Type 1 exceptional solution with $\operatorname{gcd}(x, y)=1, y>1$, then $(x, k)=\left(X_{m}, K_{m}\right)$, where

$$
X_{m}+K_{m} \sqrt{D}=\left(y^{2}+\epsilon y+1+(y+\epsilon) \sqrt{D}\right) \alpha^{m},
$$

where $m \geq 1$ and $\epsilon \pm 1$. Conversely if $\left(X_{m}, K_{m}\right)$ is given by (13.5), where $y>1$, then $\left(K_{m}, X_{m}, y\right)$ is a Type 1 exceptional solution with $\operatorname{gcd}\left(X_{m}, y\right)=1$.

PROOF. If $g=1$, formula 13.4 gives

$$
x_{n}+k_{n} \sqrt{D}=\left(y^{2}+\epsilon y+1+(y+\epsilon) \sqrt{D}\right) \alpha^{2 n} .
$$

If $g=-1$, formula 13.4 gives

$$
\begin{aligned}
x_{n}+k_{n} \sqrt{D} & =\left(y^{2}+\epsilon y+1-(y+\epsilon) \sqrt{D}\right) \alpha^{2 n} \\
& =\left(y^{2}+\epsilon y+1-(y+\epsilon) \sqrt{D}\right) \alpha^{2 n-1} \alpha \\
& =\left(y^{2}+\epsilon y+1-(y+\epsilon) \sqrt{D}\right)\left(2 y^{2}+1+2 y \sqrt{D}\right) \alpha^{2 n-1} \\
& =\left(y^{2}-\epsilon y+1+(y-\epsilon) \sqrt{D}\right) \alpha^{2 n-1} .
\end{aligned}
$$

Then (13.6) and (13.7) combine into one formula (13.5).

Conversely, formula (13.5) implies

$$
X_{m} \equiv(-1)^{m} \epsilon y \quad\left(\bmod y^{2}+1\right) .
$$

Also $X_{m}>0, K_{m}>y+1, \operatorname{gcd}\left(X_{m}, K_{m}\right)=1$ all follow by induction, using the recurrence relations

$$
\begin{aligned}
& X_{m+1}=\left(2 y^{2}+1\right) X_{m}+2 y D K_{m} \\
& K_{m+1}=\left(2 y^{2}+1\right) K_{m}+2 y X_{m} .
\end{aligned}
$$

\section{Constructing ExCeptional SOlutions}

The construction starts from the following trivial solutions:

(i) $(t, t, 0), t \geq 2$,

(ii) $\left(t, t^{2}-t+1, t-1\right), t \geq 2$,

(iii) $\left(t, t^{2}+t+1, t+1\right), t \geq 1$.

Definition 14.1. Let $(k, x, y)$ be a solution of (1.1). Then 
(i) $g_{+}(k, x, y)=(K, X, Y)$, where $Y=k$ and

$$
X+K \sqrt{k^{2}+1}=\left(x+y \sqrt{k^{2}+1}\right)\left(2 k^{2}+1+2 k \sqrt{k^{2}+1}\right) .
$$

(ii) $g_{0}(k, x, y)=(K, X, Y)$, where $Y=y$ and

$$
X+K \sqrt{y^{2}+1}=\left(x+k \sqrt{y^{2}+1}\right)\left(2 y^{2}+1+2 y \sqrt{y^{2}+1}\right) .
$$

(iii) $g_{-}(k, x, y)=(K, X, Y)$, where $Y=k$ and

$$
X+K \sqrt{k^{2}+1}=\left(x-y \sqrt{k^{2}+1}\right)\left(2 k^{2}+1+2 k \sqrt{k^{2}+1}\right) .
$$

REMARK 14.2. In all three cases $\operatorname{gcd}(X, Y)=\operatorname{gcd}(x, y)$.

LemMa 14.3. Suppose $(k, x, y)$ is an exceptional solution of (1.1). Then $g_{+}(k, x, y), g_{0}(k, x, y)$ and $g_{-}(k, x, y)$ are exceptional solutions. Morever with $T=\left(2 Y^{2}+1\right) K-2 Y X$,

(i) $g_{+}(k, x, y)=(K, X, Y)$ where $0<T<Y-1$.

(ii) $g_{0}(k, x, y)=(K, X, Y)$ where $Y+1<T$.

(iii) $g_{-}(k, x, y)=(K, X, Y)$ where $-(Y-1)<T<0$.

In all cases, we have $K>k$.

Proof. (i) We have

$$
K=2 k x+\left(2 k^{2}+1\right) y, \quad X=\left(2 k^{2}+1\right) x+\left(k^{2}+1\right) 2 k y, \quad Y=k .
$$

Taking norms in (14.1) gives $X^{2}-\left(k^{2}+1\right) K^{2}=x^{2}-\left(k^{2}+1\right) y^{2}=k^{2}$, so $X^{2}-\left(Y^{2}+1\right) K^{2}=Y^{2}$.

Also $K>2 k \geq k+1=Y+1, X>0$ and hence $(K, X, Y)$ is an exceptional solution. Next, $0<y<k-1, y=\left(2 Y^{2}+1\right) K-2 Y X=T$, so $0<T<Y-1$. Clearly $K>k$ here.

(ii) We have

$$
K=2 y x+\left(2 y^{2}+1\right) k, \quad X=\left(2 y^{2}+1\right) x+\left(y^{2}+1\right) 2 y k, \quad Y=y .
$$

Taking norms in (14.2) gives $X^{2}-\left(y^{2}+1\right) K^{2}=x^{2}-\left(y^{2}+1\right) k^{2}=y^{2}$, so $X^{2}-\left(Y^{2}+1\right) K^{2}=Y^{2}$.

Also $K>2 y^{2}+1>y+1=Y+1, X>0$ and hence $(K, X, Y)$ is an exceptional solution. Next, $y+1<k, k=\left(2 Y^{2}+1\right) K-2 Y X=T$, so $Y+1<T$. Clearly $K>k$ here.

(iii) We have

$$
K=2 k x-\left(2 k^{2}+1\right) y, \quad X=\left(2 k^{2}+1\right) x-\left(k^{2}+1\right) 2 k y, \quad Y=k .
$$

Taking norms in (14.3) gives $X^{2}-\left(k^{2}+1\right) K^{2}=x^{2}-\left(k^{2}+1\right) y^{2}=k^{2}$, so $X^{2}-\left(Y^{2}+1\right) K^{2}=Y^{2}$. Also

$$
\begin{aligned}
X & >\left(2 k^{2}+1\right) y \sqrt{k^{2}+1}-\left(k^{2}+1\right) 2 k y \\
& =y\left(2 k^{2}+1-2 k \sqrt{k^{2}+1}\right) \sqrt{k^{2}+1}>0 .
\end{aligned}
$$


We now have to prove $K>Y+1=k+1$, i.e.,

$$
2 k x>\left(2 k^{2}+1\right) y+k+1 \text {. }
$$

On squaring both sides, using $x^{2}=\left(k^{2}+1\right) y^{2}+k^{2}$, this becomes

$$
4 k^{4}>y^{2}+2\left(2 k^{2}+1\right) y(k-1)+(k+1)^{2} .
$$

However the RHS of (14.4) is $<4\left(k^{4}-2 k^{3}+2 k^{2}-k+1\right)<4 k^{4}$, if $k>1$. Hence $(K, X, Y)$ is an exceptional solution.

Finally, as $-(k-1)<-y<0$ and $y=-\left(2 Y^{2}+1\right) K+2 Y X=-T$, we have $-(Y-1)<T<0$. Also $K>k$ here.

Lemma 14.4. Let $T=\left(2 Y^{2}+1\right) K-2 Y X$.

(i) If $(K, X, Y)=g_{+}(t, t, 0)$, with $t \geq 2$, then $T=0$.

(ii) If $(K, X, Y)=g_{+}\left(t, t^{2}-t+1, t-1\right)$, with $t \geq 2$, then $T=Y-1$.

(iii) If $(K, X, Y)=g_{+}\left(t, t^{2}+t+1, t+1\right)$ with $t \geq 1$, then $T=Y+1$.

In each case $(K, X, Y)$ is an exceptional solution.

Proof. (i) $g_{+}(t, t, 0)=\left(2 t^{2}, 2 t^{3}+t, t\right)=(K, X, Y)$.

Then $K=2 t^{2}, X=2 t^{3}+t, Y=t$ and

$$
\begin{aligned}
T & =\left(2 Y^{2}+1\right) K-2 Y X \\
& =\left(2 t^{2}+1\right) 2 t^{2}-2 t\left(2 t^{3}+t\right)=0 .
\end{aligned}
$$

Also if $t \geq 2$, then $Y=t<2 t^{2}-1=K-1$, so $(K, X, Y)$ is an exceptional solution. Similarly for (ii) and (iii).

Corollary 14.5. If $x_{n}$ and $k_{n}$ are defined for $n \geq 1$ by

$$
x_{n}+k_{n} \sqrt{t^{2}+1}=t\left(2 t^{2}+1+2 t \sqrt{t^{2}+1}\right)^{n},
$$

where $t \geq 2$, then

(i) $\left(k_{1}, x_{1}, t\right)=g_{+}(t, t, 0)$.

(ii) $\left(k_{n+1}, x_{n+1}, t\right)=g_{0}\left(k_{n}, x_{n}, t\right)$.

(iii) $\left(k_{n}, x_{n}, t\right)$ is an exceptional solution for $n \geq 1$

Proof. (i) $g_{+}(t, t, 0)=\left(2 t^{2}, 2 t^{3}+t, t\right)=\left(k_{1}, x_{1}, t\right)$.

(ii) $g_{0}\left(k_{n}, x_{n}, t\right)=\left(2 t x_{n}+\left(2 t^{2}+1\right) k_{n},\left(2 t^{2}+1\right) x_{n}+\left(t^{2}+1\right) 2 t k_{n}, t\right)$

$$
=\left(k_{n+1}, x_{n+1}, t\right) \text {. }
$$

(iii) We use induction on $n \geq 1$. We know $\left(k_{1}, x_{1}, t\right)$ is an exceptional solution. Now assume $\left(k_{n}, x_{n}, t\right)$ is an exceptional solution. Then Lemma 14.3 shows that $\left(k_{n+1}, x_{n+1}, t\right)$ is also an exceptional solution.

In a similar fashion, we have 
COROLlary 14.6. If $x_{n}$ and $k_{n}$ are defined for $n \geq 1$ by

$$
x_{n}+k_{n} \sqrt{t^{2}+1}=\left(t^{2}+\epsilon t+1+(t+\epsilon) \sqrt{t^{2}+1}\right)\left(2 t^{2}+1+2 t \sqrt{t^{2}+1}\right)^{n},
$$

where $t \geq 1$ if $\epsilon=1$ and $t \geq 2$ if $\epsilon=-1$, then

(i) $\left(k_{1}, x_{1}, t\right)=g_{+}\left(t, t^{2}+\epsilon t+1, t+\epsilon\right)$.

(ii) $\left(k_{n+1}, x_{n+1}, t\right)=g_{0}\left(k_{n}, x_{n}, t\right)$.

(iii) $\left(k_{n}, x_{n}, t\right)$ is an exceptional solution for $n \geq 1$.

REMARK 14.7. Recall that an exceptional solution $(k, x, y)$ is of Type 1 , if $y^{2}+1$ divides $x+y$ or $x-y$. Any other exceptional solution is called Type 2. Then we proved in Theorem 9.1 that the exceptional solutions $\left(k_{n}, x_{n}, t\right)$ in Corollary 14.5 are the $(k, x, y)$ for which $y$ divides $x$ and $y>1$ and that these are Type 1 solutions. Contrastingly, we proved in Corollary 13.2 that those in Corollary 14.6 are the Type 1 exceptional solutions $(k, x, y)$ for which $\operatorname{gcd}(x, y)=1$.

LEMMA 14.8. (i) Suppose that $(k, x, y)$ is an exceptional solution. Then $g_{+}(k, x, y)$ and $g_{-}(k, x, y)$ are Type 2 exceptional solutions.

(ii) Suppose that $(k, x, y)$ is a Type 2 exceptional solution. Then $g_{0}(k, x, y)$ is a Type 2 exceptional solution.

Proof. (i) $g_{+}(k, x, y)$ and $g_{-}(k, x, y)$ have the form $(K, X, k)$, with $X=$ $R x+e D S y, e= \pm 1$. We have to prove that $X \pm k$ are not divisible by $k^{2}+1$.

$$
\begin{array}{ll}
X-k=R x+e D S y-k \equiv-x-k & \left(\bmod k^{2}+1\right), \\
X+k=R x+e D S y+k \equiv-x+k & \left(\bmod k^{2}+1\right) .
\end{array}
$$

Also $x<k^{2}-k+1$, as $y<k-1$. Also $x \neq k$ here. So

$$
0<|x-k|<x+k<k^{2}+1
$$

and neither $x-k$ nor $x+k$ is divisible by $k^{2}+1$.

(ii) Suppose $(k, x, y)$ is a Type 2 exceptional solution. Then

$$
\begin{aligned}
g_{0}(k, x, y) & =\left(2 y x+\left(2 y^{2}+1\right) k,\left(2 y^{2}+1\right) x+\left(y^{2}+1\right) 2 y k, y\right) \\
& =(K, X, Y) .
\end{aligned}
$$

Also $Y=y$ and

$$
\begin{aligned}
X \pm Y & =\left(2 y^{2}+1\right) x+\left(y^{2}+1\right) 2 y \pm y \\
& \equiv-x \pm y \not \equiv 0 \quad\left(\bmod y^{2}+1\right) .
\end{aligned}
$$




\section{THE RECURSIVE CONSTRUCTION}

In the previous section, we have established the following. Let $\mathscr{E}$ be the set of exceptional solutions $(k, x, y)$. Then with $R=2 Y^{2}+1, S=2 Y$ and $T=R K-S X$,

(i) $g_{0}$ maps $\mathscr{E}$ one-to-one into $\{(K, X, Y) \in \mathscr{E} \mid Y+1<T\}$.

(ii) $g_{+}$maps $\mathscr{E}$ one-to-one into $\{(K, X, Y) \in \mathscr{E} \mid 0<T<Y-1\}$.

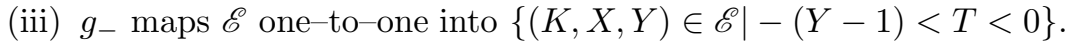

(iv) $g_{+}$maps $\{(t, t, 0) \mid t \geq 2\}$ one-to-one into $\{(K, X, Y) \in \mathscr{E} \mid T=0\}$.

(v) $g_{+} \operatorname{maps}\left\{\left(t, t^{2}-t+1, t-1\right) \mid t \geq 2\right\}$ one-to-one into $\{(K, X, Y) \in$ $\mathscr{E} \mid T=Y-1\}$.

(vi) $g_{+}$maps $\left\{\left(t, t^{2}+t+1, t+1\right) \mid t \geq 1\right\}$ one-to-one into $\{(K, X, Y) \in$ $\mathscr{E} \mid T=Y+1\}$.

It is easy to check that these mappings are surjective.

We construct a forest of exceptional solutions, as follows. We start from an exceptional solution obtained by applying $g_{+}$to each of the trivial solutions (a) $(t, t, 0), t \geq 2$, (b) $\left(t, t^{2}-t+1, t-1\right), t \geq 2$, (c) $\left(t, t^{2}+t+1, t+1\right), t \geq 1$. Then recursively, from an exceptional solution $(k, x, y)$, we produce three further exceptional solutions.

Because of Remark 14.2, the solutions in trees with root node (a) will have $\operatorname{gcd}(x, y)=t \geq 2$, while those with root node (b) or (c), will have $\operatorname{gcd}(x, y)=1$.

Figures 1-3 give fragments of the forest of exceptional solutions. We

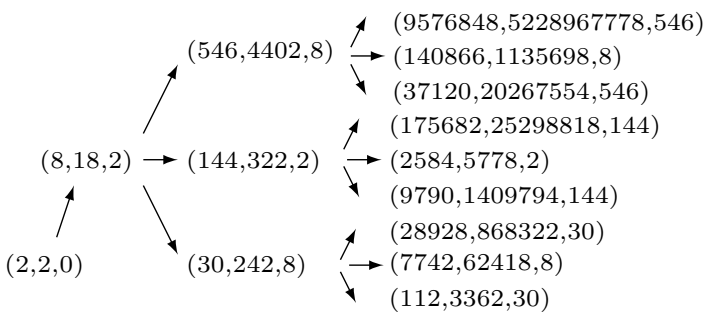

Figure 1. Tree fragment starting from $(t, t, 0)=(2,2,0)$.

now show that all exceptional solutions occur in the forest and are reached by a unique path from a root node.

LEMMA 15.1. If $(K, X, Y)$ is an exceptional solution and $T=R K-S X$ where $R=2 Y^{2}+1$ and $S=2 Y$, then

(a) $-(Y-1)<T$,

(b) $T \neq Y$. 


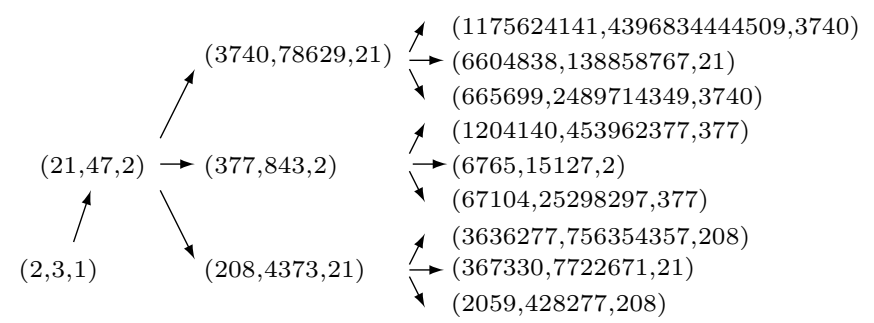

Figure 2. Tree fragment starting from $\left(t, t^{2}-t+1, t-1\right)=(2,3,1)$.

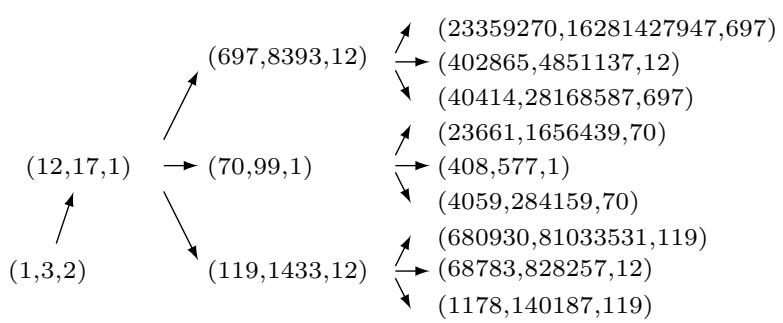

Figure 3 . Tree fragment starting from $\left(t, t^{2}+t+1, t+1\right)=(1,3,2)$.

Proof. First we prove (a).

$$
\begin{aligned}
& -(Y-1)<T \Longleftrightarrow-T=S X-R K<Y-1 \\
& \Longleftrightarrow 2 Y X<\left(2 Y^{2}+1\right) K+Y-1 \\
& \Longleftrightarrow 4 Y^{2} X^{2}<\left(4 Y^{4}+4 Y^{2}+1\right) K^{2}+2 K\left(2 Y^{2}+1\right)(Y-1)+(Y-1)^{2} \\
& \Longleftrightarrow 4 Y^{4}<K^{2}+2 K\left(2 Y^{2}+1\right)(Y-1)+(Y-1)^{2} .
\end{aligned}
$$

However, the last inequality follows from $K>Y+1$.

(b) Now assume $T=Y$. Then $\left(2 Y^{2}+1\right) K-2 Y X=Y$, so $Y$ divides $K$.

Hence $Y$ divides $X$. Let $K=Y W$ and $X=Y Z$. Then

$$
\begin{aligned}
\left(2 Y^{2}+1\right) W-2 Y Z & =1, \\
Z^{2}-\left(Y^{2}+1\right) W^{2} & =1 .
\end{aligned}
$$

Eliminating $Z$ gives $4 Y^{2}(W+1)=(W-1)^{2}$. Hence $W$ is odd, $W=2 U+1$ and $2 Y^{2}(U+1)=U^{2}$. Then $U+1$ divides $U^{2}$, which contradicts $\operatorname{gcd}\left(U+1, U^{2}\right)=$ 1.

Definition 15.2. Let $(K, X, Y)$ be an exceptional solution. Let $R=$ $2 Y^{2}+1, S=2 Y, D=Y^{2}+1$ and $T=R K-S X$. Then

$$
h(K, X, Y)= \begin{cases}g_{0}^{-1}(K, X, Y) & \text { if } Y+1<T, \\ g_{+}^{-1}(K, X, Y) & \text { if } 0 \leq T \leq Y+1, T \neq Y, \\ g_{-}^{-1}(K, X, Y) & \text { if }-(Y-1)<T<0 .\end{cases}
$$


REMARK 15.3. By virtue of Lemma $15.1, h$ is well-defined and $h(K, X, Y)$ $=(k, x, y)$ is either an exceptional solution with $k<K$, or one of the trivial solutions $\left(Y, Y^{2}+\epsilon Y+1, Y+\epsilon\right)$ or $(Y, Y, 0)$.

It follows that repeated application of $h$ on an exceptional solution $(K, X, Y)$ will eventually reach a trivial solution $(k, x, y)$ and consequently $(K, X, Y)$ occurs in the tree whose root node is $(k, x, y)$. As the path from $(K, X, Y)$ back to a root node is uniquely defined, the forest of exceptional solutions contains every exceptional solution just once. Dujella's conjecture means that no two nodes can have the same $K$.

The forest can be used to check that Dujella's conjecture holds for all $k$ not exceeding a given bound. For as one travels along a path from a root node, the value of $k$ increases; also as $t$ is increased in one of the three types of root node $(k, x, y)$, so does the size of $K$, where $g_{+}(k, x, y)=(K, X, Y)$.

It is clear by induction that the exceptional solutions have the form $(K(t), X(t), Y(t))$, where the components are polynomials with integer coefficients, corresponding to the three types of root node:

$$
(t, t, 0), t \geq 2 ;\left(t, t^{2}-t+1, t-1\right), t \geq 2 ;\left(t, t^{2}+t+1, t+1\right), t \geq 1 .
$$

\section{FAMILIES OF $k$ WITH EXPLICIT EXCEPTIONAL SOLUTIONS}

The following examples were suggested by an extension of Table 1 to $k \leq 2^{32}$. We use the terminology of Proposition 2.1. The continued fraction identities were proved using formula (iv) of Lemma 6.2.

EXAmPLE 16.1. $g_{+}(t, t, 0)=(k, x, y)=\left(2 t^{2}, 2 t^{3}+t, t\right), t \geq 2$. Then

$$
d=t, a=2 t^{2}+2 t+1, b=2 t^{2}-2 t+1, p=1, q=1 .
$$

Then

$$
\sqrt{b / a}=[0,1, \overline{t-1,1,1, t-1,2}], \text { period length } 5 .
$$

Also $Q_{2}=4 t=2 k / d$ and $p / q=A_{1} / B_{1}$.

\begin{tabular}{c|cccc}
\hline$t$ & 2 & 3 & 4 & 5 \\
\hline$k$ & 8 & 18 & 32 & 50 \\
\hline
\end{tabular}

EXAMPLE 16.2. $g_{0} g_{+}(t, t, 0)=(k, x, y)=\left(8 t^{4}+4 t^{2}, 8 t^{5}+8 t^{3}+t, t\right)$, where $t \geq 2$. Then

$d=t, a=8 t^{4}+8 t^{3}+8 t^{2}+4 t+1, b=8 t^{4}-8 t^{3}+8 t^{2}-4 t+1, p=1, q=1$.

Then

$\sqrt{b / a}=[0,1, \overline{t-1,1,1, t-1,1,1, t-1,1,1, t-1,2}]$, period length 11.

Also $Q_{2}=16 t^{3}+8 t=2 k / d$ and $p / q=A_{1} / B_{1}$.

\begin{tabular}{c|cccc}
\hline$t$ & 2 & 3 & 4 & 5 \\
\hline$k$ & 144 & 684 & 2112 & 5100 \\
\hline
\end{tabular}


ExAmPLE 16.3. $g_{+}\left(t, t^{2}+t+1, t+1\right)=(k, x, y), t \geq 1$. Then

$$
k=4 t^{3}+4 t^{2}+3 t+1, \quad x=4 t^{4}+4 t^{3}+5 t^{2}+3 t+1, y=t
$$

and $d=1$ if $t$ is odd, whereas $d=2$ if $t$ is even.

$$
\begin{aligned}
& a= \begin{cases}\left(4 t^{4}+8 t^{3}+9 t^{2}+6 t+2\right) / 2 & \text { if } t \text { is even, } \\
4 t^{4}+8 t^{3}+9 t^{2}+6 t+2 & \text { if } t \text { is odd. }\end{cases} \\
& b= \begin{cases}8 t^{2}+2 & \text { if } t \text { is even, } \\
4 t^{2}+1 & \text { if } t \text { is odd. }\end{cases}
\end{aligned}
$$

Then $p=1$ and $q=t / 2$ if $t$ is even, whereas $q=t$ if $t$ is odd.

(i) If $t$ is even,

$$
\sqrt{b / a}=[0, t / 2, \overline{1,1, t-1,1,1, t-1,1,1, t}] \text {, period length } 9 .
$$

Also $Q_{2}=4 t^{3}+4 t^{2}+3 t+1=k=2 k / d$ and $p / q=A_{1} / B_{1}$.

(ii) If $t$ is odd,

$$
\sqrt{b / a}=[0, t+1, \overline{2 t, 2 t, 2 t+2}] \text {, period length } 3 .
$$

Also $Q_{1}=b=4 t^{2}+1, Q_{2}=4 t^{2}+4 t+1, P_{2}=4 t^{3}+4 t^{2}+t+1$. Hence $Q_{2}-Q_{1}+2 P_{2}=2 k$ and $p / q=\left(A_{1}-A_{0}\right) /\left(B_{1}-B_{0}\right)$.

\begin{tabular}{c|ccccc}
\hline$t$ & 1 & 2 & 3 & 4 & 5 \\
\hline$k$ & 12 & 55 & 154 & 333 & 616 \\
\hline
\end{tabular}

17. The unicity ConjeCture implies the $D(-1)$ CONJECture

The $D(-1)$ quadruples conjecture states that there do not exist four distinct positive integers such that the product of any two is one plus a square. The following argument was supplied by Andrej Dujella. Assume that the unicity conjecture is true and let $a, b, c, d$ be a $D(-1)$-quadruple with $0<a<b<c<d$. Then $a=1$ by [3] and hence

$$
b=r^{2}+1, c=s^{2}+1, d=t^{2}+1 .
$$

Now consider the equation $\left(y^{2}+1\right)\left(t^{2}+1\right)=x^{2}+1$, i.e.,

$$
x^{2}-\left(t^{2}+1\right) y^{2}=t^{2} \text {. }
$$

By the unicity conjecture, this diophantine equation has at most one solution with $0<y<t-1$.

But by assumption, it has at least two solutions with $0<y<t$, namely, $y=r$ and $y=s$, and hence we must have $s=t-1$.

However this contradicts a gap property [3, Lemma 9] which implies that $d>c^{2}$. For the inequality

$$
d=t^{2}+1>c^{2}=\left((t-1)^{2}+1\right)^{2}
$$

does not hold for any $t>2$. 


\section{ACKNOWLEDGEMENTS.}

The authors thank Alan Offer for valuable discussions concerning the construction of the forest. Also the manuscript has been greatly improved by Professor Andrej Dujella and the referees.

\section{REFERENCES}

[1] L. E. Dickson, Introduction to the theory of numbers, Dover Publications, 1957.

[2] A. Dujella, Continued fractions and RSA with small secret exponent, Tatra Mt. Math. Publ. 29 (2004), 101-112.

[3] A. Dujella and C. Fuchs, Complete solution of a problem of Diophantus and Euler, J. London Math. Soc. 71 (2005), 33-52.

[4] A. Dujella and B. Jadrijević, A family of quartic Thue inequalities, Acta Arith. 111 (2004), 61-76.

[5] A. Filipin, Y. Fujita and M. Mignotte, The non-extendibility of some parametric families of D(-1)-triples, Q. J. Math. 63 (2012), 605-621.

[6] O. Perron, Die Lehre von den Kettenbrüchen,Teubner, Stuttgart, 1954.

[7] P. Z. Yuan and Z. F. Zhang, On the diophantine equation $X^{2}-\left(1+a^{2}\right) Y^{4}=-2 a$, Sci. China Math. 53 (2010), 2143-2158.

K. R. Matthews

Department of Mathematics

University of Queensland

Brisbane, Australia, 4072

and

Centre for Mathematics and its Applications

Australian National University

Canberra, ACT

Australia, 0200

E-mail: keithmatt@gmail.com

J. P. Robertson

Actuarial and Economic Services Division

National Council on Compensation Insurance

Boca Raton, FL 33487

USA

E-mail: jpr2718@gmail.com

J. White

14 Nash Place, Stirling

Canberra, ACT

Australia, 2611

E-mail: mathimagics@yahoo.co.uk

Received: 3.11.2012.

Revised: 8.2.2013. 